\title{
Lectin Array Analysis of Purified Lipooligosaccharide: A Method for the Determination of Molecular Mimicry
}

Evgeny A Semchenko, Marc Moutin, Victoria Korolik, Joe Tiralongo and Christopher J Day*

Institute for Glycomics, Griffith University, Gold Coast, Queensland, Australia

\begin{abstract}
Surface glycosylation of bacteria is involved in many critical host-microbe interactions. Lectin arrays consisting of diverse carbohydrate binding proteins have proven to be an important tool for evaluating a wide variety of glycosylation, including that present on whole bacteria. However, assessing glycosylation on whole bacteria using lectin arrays may not reflect bacterial glycosylation, but interactions between bacteria and the glycosylation present on lectins. The lipooligosaccharide of Campylobacter jejuni NCTC11168 and 81-176 are known to mimic the human monosialylated gangliosides. This molecular mimicry by $C$. jejuni can result in the post infection sequelae Guillain-Barré syndrome. Using C. jejuni as a model system and a discreet lectin and antibody array, a method, applicable to many organisms has been developed and validated by to screening of the purified lipooligosaccharide of $C$. jejuni for molecular mimicry to monosiylated gangliodises. In case of $C$. jejuni, knowing whether clinically important bacterial strains are capable of inducing severe autoimmune responses may aid in prevention and/or early diagnosis of debilitating post infection conditions.
\end{abstract}

Keywords: Lipooligosaccharide; Glycosylation; Lectin arrays

\section{Introduction}

Bacterial surface glycosylation is involved in many critical and diverse host-microbe interactions including adherence and immune modulation [1]. The analysis of bacterial surface glycosylation traditionally has been performed using nuclear magnetic resonance (NMR) spectroscopy, mass spectrometry (MS) and blotting based techniques [2-7]. Both NMR spectroscopy and MS are powerful techniques, but require relatively large quantities of highly purified glycan for structural analysis. Blotting methodologies, such as lectin blotting [7], have the advantage of only requiring small amounts of partially purified glycosylated protein or lipid for analysis, however only a single lectin interaction can be assessed per blot and the results can be ambiguous due to the difficulties in ensuring equimolar loading of test compounds into individual wells on the gel. In all cases these methodologies only provide low-throughput capabilities.

Lectin arrays consisting of diverse carbohydrate binding proteins covalently immobilised on glass microarray slides, have proven to be an important tool for evaluating cell surface glycosylation on whole bacteria and eukaryotic cells [1,8-10], eliminating the need to purify surface glycoproteins and lipids prior to analysis. However, assessing glycosylation on whole bacteria using lectin arrays may not be an accurate reflection of the bacterial glycosylation. Lectins are glycoproteins [11,12], therefore when immobilized on microarray slides they may themselves act as receptors for bacterial carbohydrate binding proteins. That is, bacterial glycan recognising adhesins [1317] may interact with carbohydrate structures present on immobilised lectins rather than lectins recognising bacterial glycoconjugates. In order to overcome this potentially significant limitation, we report here the analysis of semi-purified bacterial glycan using a discrete lectin array that requires only a small quantity of product for accurate, quick, and reproducible glycan structure determination. As the model systems for lectin array-based glycan structure determination we assessed both purified and crudely isolated C. jejuni lipooligosaccharide (LOS).

Campylobacter jejuni is a Gram-negative spiral rod bacteria that synthesizes both N-linked and O-linked glycans [18]. The strains of C. jejuni with that produce sialylated LOS (biosynthesis cluster types $\mathrm{A}, \mathrm{B}, \mathrm{C}, \mathrm{M}$ and $\mathrm{R}$ ) are capable of causing an autoimmune response against human gangliosides. This autoimmune response occurs in approximately 1:1000 people infected with $C$. jejuni leading to the development of the debilitating and life-threatening diseases GuillainBarré syndrome (GBS) or Miller Fisher Syndrome (MFS) [19-21]. Our novel approaches to glycan structure identification permitted the elucidation of a clinically important ganglioside structures involved in molecular mimicry, the basis for the development of GBS.

\section{Materials and Methods}

\section{Bacterial strains and growth conditions}

The original isolate of C. jejuni NCTC 11168 (11168-O) previously characterized [22] was kindly supplied by D.J. Newell (Veterinary Laboratories Agency, Weybridge, UK). The human isolates C. jejuni 81-176 was donated by James G. Fox (Massachusetts Institute of Technology, Cambridge, Massachusetts, USA) and C. jejuni 224 was obtained from the Royal Melbourne Institute of Technology (Melbourne, Vic., Australia). C. jejuni were grown on blood agar, composed of Columbia agar containing $5 \%$ (v/v) defibrinated horse blood and Skirrow's antibiotic supplement (Oxoid), under microaerobic conditions $\left(5 \% \mathrm{O}_{2}, 10 \% \mathrm{CO}_{2}\right.$ and $\left.85 \% \mathrm{~N}_{2}\right)$ at $37^{\circ} \mathrm{C}$ for $48 \mathrm{~h}$.

\section{Lipooligosaccharide preparations}

Crude LOS preparation: Blood agar-grown bacteria were harvested in $1 \mathrm{~mL}$ of sterile water, washed once in $1 \mathrm{~mL}$ of sterile water, and lysed by heating. Prior to lysis, samples were adjusted for bacterial number by $\mathrm{OD}_{600}$ measurement of bacterial suspensions. Mini-preparations of LOS were prepared by treating the whole-cell extracts with proteinase

*Corresponding author: Christopher J Day, Institute for Glycomics, Griffith University Gold Coast Campus, Griffith University, Australia, Tel: +61-7-55529351; Fax: +61-7-55528098; E-mail: c.day@griffith.edu.au

Received February 20, 2011; Accepted April 30, 2011; Published Septembet 29, 2011

Citation: Semchenko EA, Moutin M, Korolik V, Tiralongo J, Day CJ (2011) Lectin Array Analysis of Purified Lipooligosaccharide: A Method for the Determination of Molecular Mimicry. J Glycom Lipidom 1:105. doi:10.4172/2153-0637.1000105

Copyright: (C) 2011 Semchenko EA, et al. This is an open-access article distributed under the terms of the Creative Commons Attribution License, which permits unrestricted use, distribution, and reproduction in any medium, provided the original author and source are credited. 
$\mathrm{K}$ as described previously [23]. The LOS mini-preparations from single colonies were prepared by collecting and washing cells in $40 \mu \mathrm{L}$ of sterile water followed by heat lysis. These preparations were diluted 10 fold prior to gel electrophoresis or lectin array analysis.

Purified LOS preparation: C. jejuni LOS was purified by subjecting the C. jejuni cell biomass to hot phenol-water treatment using $90 \%$ (v/v) aqueous phenol at $65^{\circ} \mathrm{C}$ for $10 \mathrm{~min}$ [24], followed by enzymatic treatment as previously described [25]. The LOS preparations were adjusted to $15 \mu \mathrm{g} / \mu \mathrm{L}$ with distilled water prior to gel electrophoresis or lectin array analysis.

\section{Electrophoretic analyses}

Equal quantities of either LOS mini-preparations or purified LOS $(\sim 15 \mu \mathrm{g})$ was resolved on SDS-PAGE $(5.5 \%(\mathrm{w} / \mathrm{v})$ and $10 \%(\mathrm{v} / \mathrm{v})$ stacking and separating acrylamide gels, respectively) containing $6 \mathrm{M}$ urea and $0.3 \mathrm{mM}$ tricine (Tricine-SDS-PAGE) as previously described [26]. Following electrophoresis at $20 \mathrm{~V}$ for $1 \mathrm{~h}$ to maximize stacking and then at $200 \mathrm{~V}$ for $30 \mathrm{~min}$, gels were fixed and carbohydrate bands visualised by silver staining [27].

\section{Lectin and western blotting}

In addition to silver staining, fractionated C. jejuni LOS was electrotransferred from Tricine SDS-PAGE gels to Pall ${ }^{\mathbb{B}}$ PVDF membranes using a Trans-Blot SD Semi-Dry Transfer Cell (Bio-Rad) at $30 \mathrm{~V}$ for $60 \mathrm{~min}$. Membranes were subsequently probed using either

\begin{tabular}{|l|l|l|}
\hline Primer Name & Primer Sequence & $\mathrm{Tm}$ \\
\hline ORF6 F & GTAGTAGATGATTTGGGTAATGATAAA & $47^{\circ} \mathrm{C}$ \\
\hline ORF6 R & ATAGAATTGCTATTTACATGCTGG & \\
\hline ORF8 F & CCTTTGATAATCCCTGAAATAGGT & $50^{\circ} \mathrm{C}$ \\
\hline ORF8 R & TCCTTTGCACTTATACCACCTT & \\
\hline ORF5/10 F & GGTGTTATAGGATATAATGATTGTACTGATGG & $49^{\circ} \mathrm{C}$ \\
\hline ORF5/10 R & CCTCTGTTGTATCTATATCCAAACTAGC & \\
\hline ORF12 F & GCCACAACTTTCTATCATAATCCCGC & $50^{\circ} \mathrm{C}$ \\
\hline ORF12 R & CGCCATAACTCAAACGCTCATCTATT & \\
\hline ORF14 F & GCTAGAACACCCTAAAGTGACTAA & $47^{\circ} \mathrm{C}$ \\
\hline ORF14 R & TGGCACTAAATTGTAATAAATGGC & \\
\hline ORF16 F & AGATCTGCAGCGTTTAGTGATTATTTTAG & $47^{\circ} \mathrm{C}$ \\
\hline ORF16 R & TACTCCAGATCCCCTATCGTCTC & \\
\hline
\end{tabular}

Table 1: Primers used in this study.

\begin{tabular}{|c|c|c|c|c|c|}
\hline $\begin{array}{l}\text { Lectin/ } \\
\text { Antibody }\end{array}$ & Specificity & $11168[P]$ & $11168[\mathrm{C}]$ & $81-176$ & 224 \\
\hline CTB & $\mathrm{G}_{\mathrm{M} 1}>\mathrm{G}_{\mathrm{M} 2}>\mathrm{G}_{\mathrm{M} 3}$ & - & - & - & - \\
\hline PNA & Galß1-3GalNAc & +++ & +++ & + & + \\
\hline LFA & Neu5Ac & + & + & ++ & ++ \\
\hline MAA & a2-3Neu5Ac & + & + & + & + \\
\hline Anti- $G_{M 2}$ & $\mathrm{G}_{\mathrm{M} 2}$ & + & + & ++ & - \\
\hline Anti-G $\mathrm{M}_{\mathrm{M}}$ & $\mathrm{G}_{\mathrm{M} 1}$ & +++ & +++ & - & +++ \\
\hline Jacalin & $\alpha-D-G a l$ and Galß1-3GalNAc & ++ & ++ & + & + \\
\hline ConA & $\alpha-D-M a n>\alpha-D-G l c$ & - & - & - & - \\
\hline VAA & $\beta-D-G a l$ & +++ & +++ & + & + \\
\hline DBA & Terminal $\alpha-D-G a I N A c$ & - & - & - & - \\
\hline
\end{tabular}

-: No binding observed

+: Binding observed at lectin print concentration of $500 \mu \mathrm{g} / \mathrm{mL}$ (lectin) or 1:100 diltution (antibody)

++: Binding observed at lectin print concentration of $250 \mu \mathrm{g} / \mathrm{mL}$ (lectin) or 1:1000 diltution (antibody).

+++ : Binding observed at lectin print concentration of $125 \mu \mathrm{g} / \mathrm{mL}$ (lectin) or 1:10000 diltution (antibody).

Table 2: Results of the lectin array of phenol purified and crudely prepared LOS from C. jejuni strains NCTC11168, 81-176 and 224.

A
\begin{tabular}{|l|l|l|l|l|l|l|l|l|}
\hline Strain & 6 & 8 & $5 \backslash 10$ & 12 & 14 & 16 & Type & Known Type \\
\hline 224 & + & + & - & + & - & + & R & N/A \\
\hline 11168 & + & + & + & + & + & + & C & C \\
\hline $81-176$ & + & + & - & + & - & - & A/B & B \\
\hline
\end{tabular}

B

Cluster

\begin{tabular}{|l|l|l|l|l|l|l|l|l|l|l|l|l|l|}
\hline $\mathbf{A}$ & 1 & 2 & 3 & 4 & 5 & 6 & 7 & 8 & 9 & 10 & 11 & 12 & 13 \\
\hline
\end{tabular}

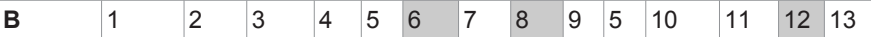

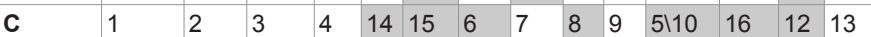

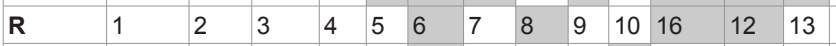

\begin{tabular}{|l|l|l|l|l|l|l|l|l|l|l|l|l|l|l|}
\hline $\mathbf{M}$ & 1 & 2 & 51 & 3 & 7 & 8 & 9 & 10 & 11 & 12 & 13 & & & \\
\hline
\end{tabular}

Table 3: LOS Biosynthesis cluster analysis. A). Cluster types can be differentiated based on the presence of ORFs within the genome. C. jejuni strains 11168 and 81 . 176 were known due to the availability of genome sequences and were confirmed by PCR to be class $C$ and A/B respectively. Strain 224 was found to be class R. B). Biosynthesis clusters capable of ganalioside molecular mimicry through production of sialylated LOS.

horseradish peroxidase (HRP)-conjugated CTB (Cholera toxin subunit B) $(3 \mu \mathrm{g} / \mathrm{mL}), \mathrm{HRP}$-conjugated PNA (Peanut Agglutinin) $(5 \mu \mathrm{g} / \mathrm{mL})$, or HRP-conjugated anti-GM ganglioside IgG (diluted 1:3000) in PBS. Membranes were developed SuperSignal West Pico Chemiluminescent Substrate (Thermo Scientific) according to the manufacturer's instructions.

\section{LOS biosynthesis cluster typing.}

LOS biosynthesis clusters were typed using a PCR methodology based upon the published gene content of the LOS gene clusters $[28,29]$. Typing was performed using the primers and at annealing temperatures presented in (Table 1). Reactions were performed using 10 pmoles of forward and reverse primer, $200 \mu \mathrm{M}$ dNTPs, using the Phusion DNA Polymerase system (Finnzymes) to a final volume of 50 $\mu \mathrm{L}$.

\section{Lectin array preparation}

Lectins were purchased from EY Laboratories and Sigma-Aldrich, and anti-ganglioside antibodies were purchased from Matreya (Table 2). Four identical sub-arrays per slide were printed essentially as previously described [30,31] onto epoxy functionalised glass slides (SuperEpoxy II; ArrayIt) at three different lectin concentrations (500 $\mu \mathrm{g} /$ $\mathrm{mL}, 250 \mu \mathrm{g} / \mathrm{mL}$ and $125 \mu \mathrm{g} / \mathrm{mL}$ ), and anti-ganglioside antibodies were printed at 1:100 to 1:10,000 dilutions across a 10-fold serial dilution. Printed slides were subsequently neutralised using ethanolamine as previously described [31]. Lectins and antibodies printed on our array and their published specificities are presented in Table 2.

\section{Lectin array analysis of $C$. jejuni LOS and whole $C$. jejuni}

Purified and crudely isolated LOS was labelled using the lipophillic dye BODIPY ${ }^{\circledR}$ TR methyl ester (BODIPY; Invitrogen). One $\mathrm{mM}$ BODIPY $(6 \mu \mathrm{L})$ was added to $5 \mu$ g of LOS in 1x PBS containing $1 \mathrm{mM}$ $\mathrm{CaCl}_{2}$ and $1 \mathrm{mM} \mathrm{MgCl}$ to a final volume of $30 \mu \mathrm{L}$ and incubated at room temperature for $15 \mathrm{~min}$ prior to direct application to printed lectin arrays. Subarrays were separated using $25 \mu \mathrm{L}$ gene frames (Thermo Scientific). LOS was incubated on the arrays for 15 minutes, followed by 3 washes with $1 x$ PBS containing $1 \mathrm{mM} \mathrm{CaCl}$ and $1 \mathrm{mM}$ $\mathrm{MgCl}_{2}$. Controls for BODIPY and unlabelled LOS were also applied to the array and washed in the same way. Whole $C$. jejuni was labelled using BODIPY by the addition of $1 \mathrm{mM}$ BODIPY to $10^{8} \mathrm{CFU}$ of $C$. jejuni in $1 \mathrm{xPBS}$ and incubated at $37^{\circ} \mathrm{C}$ for 30 mins. Application of $C$. jejuni to the arrays and washing was performed as previously described [30].s Image acquisition and data processing was performed using 
the ProScanArray Microarray 4-Laser Scanner and the ProScanArray imaging software ScanArray Express from PerkinElmer as previously described [30]. Analysis was limited to presence or absence of binding to lectin spots across a 1:2 serial dilution rather then absolute binding levels. All positive reported binding are spots that were both visible by visual inspection of the array and significantly above back ground by T-test in Microsoft Excel. Lectin arrays were performed a minimum of twice per LOS sample.

\section{Results}

The LOS of C. jejuni strain NCTC 11168 is known to mimic the human ganglioside structure, $\mathrm{G}_{\mathrm{Ml}}$, and was therefore chosen to evaluate the viability of screening both phenol purified and crudely prepared LOS samples using our discrete lectin array. Initial analysis of the two alternatively isolated LOS samples from C. jejuni NCTC 11168 was performed using traditional SDS-PAGE silver staining and lectin blotting analysis (Figure 1). Silver staining revealed no differences in electrophoretic mobility between the phenol purified and crudely isolated LOS preparations (Figure 1A). Lectin blotting using HRP-conjugated PNA and CTB also revealed no differences in binding ability between sample preparations, with as expected [6] significant binding of both lectins (Figure $1 \mathrm{~B}$ and $1 \mathrm{C}$ respectively). Taken together these data show that both LOS preparations have similar glycoconjugate components, specifically LOS that mimics $\mathrm{G}_{\mathrm{M1}}$ (as identified through PNA and CTB positive binding), and hence both LOS preparations provide an excellent tool for assessment of lectin array technology. Due to the fact that the $C$. jejuni LOS structure with molecular mimicry with human gangliosides been reported [6,3234 ], lectins were selected to generate a tailored array that would most effectively identify the individual carbohydrate motifs of structures containing monosialylganglioside mimicry (Table 2). The lectins selected included those known to bind the $\mathrm{G}_{\mathrm{M} 1}$ mimic $C$. jejuni $\mathrm{NCTC}$ 11168 LOS (PNA and CTB) $[6,34,35]$, and those assumed to bind based on the published structures of NCTC11168 and 81-176 [6,32],

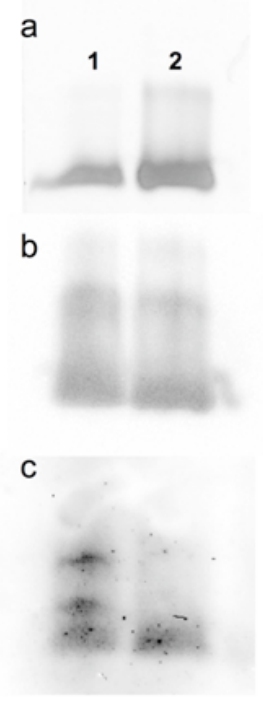

Figure 1: Silver-stained SDS-PAGE gel and Lectin blots of Crude and Phenol purified LOS preparations extracted from $C$. jejuni NCTC 11168. A). Silver stained LOS preparations. B). CTB blotting result for LOS preparations. C). PNA blotting result for LOS preparations. Lane 1 in each pane is Crude LOS preparation, while lane 2 is the phenol purified preparation.

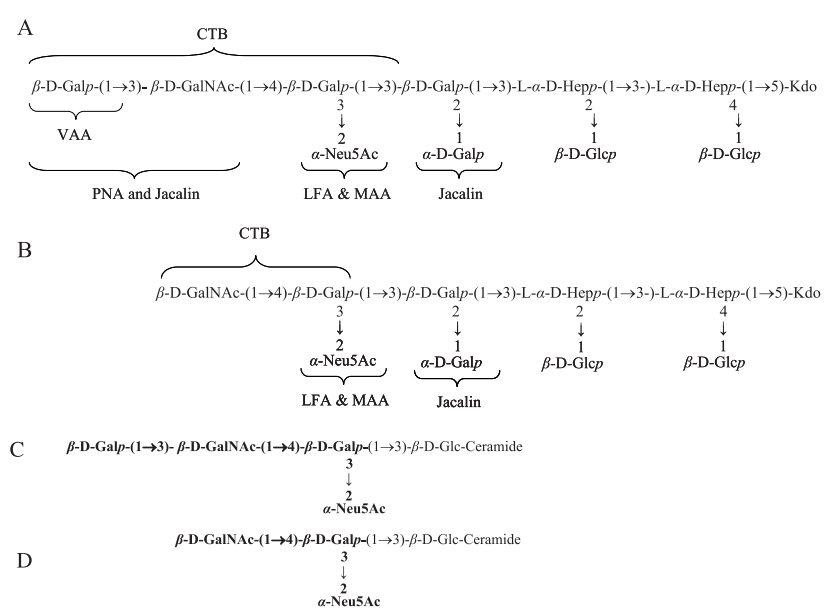

Figure 2: Published structures of the LOS of $C$. jejuni NCTC 11168, 81176 and human $\mathrm{G}_{\mathrm{M} 1}$ and $\mathrm{G}_{\mathrm{M} 2}$. A). C. jejuni NCTC 11168 [6] and the expected binding for lectins/antibodies present on the array. B). C. jejuni 81-176 [32] and the expected binding for lectins/antibodies present on the array. N.B. Expected lectin binding is predicted from specificities listed in Table 2. Lower specificity interactions are also possible. Presented structures are the published majority structure for each strain [6,32]. C). Structure of $G_{M 1}$ (bolded sugar residues are identical to published $C$. jejuni 11168 structure shown in A). D). Structure of $G_{M 2}$ (bolded sugar residues are identical to published 81-176 structure shown in $\mathbf{B}$ ).

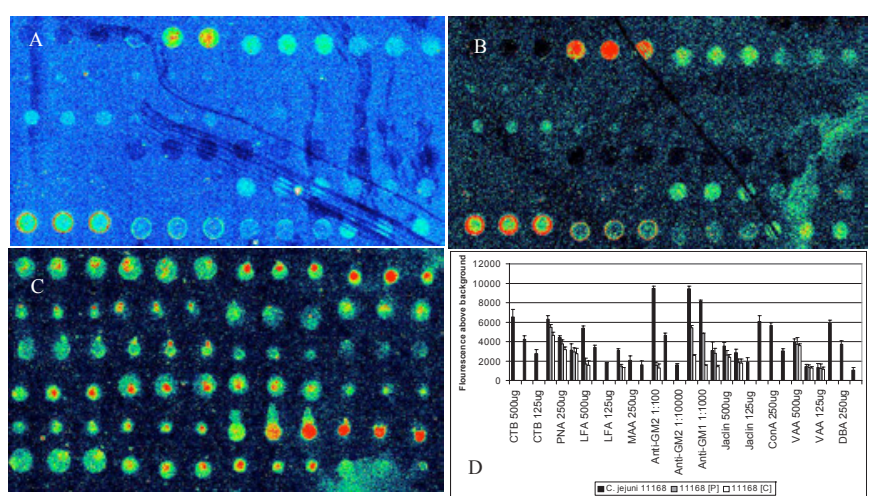

Figure 3: Analysis of live C. jejuni 11168 compared to purified LOS. A). Lectin array analysis of crude LOS extract prepared from C. jejuni 11168. B). Lectin array analysis of phenol purified LOS extract prepared from C. jejuni 11168. C). Lectin array analysis of live C. jejuni 11168. D). Graphical representation of the array analysis. Units are fluorescence above background. Error bars equal one standard error of the mean. Any data not significantly different from background has been set to zero units. Lectins are listed in same order as Table 2.

LFA (Limax flavus agglutinin), MAA (Maackia amurensis agglutinin), VAA (Viscum album agglutinin) and Jacalin (Jackfruit; Artocarpus integrifolia). Both DBA (Dolichos biflorus agglutinin) and ConA (Canavalia ensiformis agglutinin) were used as negative controls due to the absence of terminal GalNAc (recognised by DBA) and Man/ GlcNAc (recognised by ConA) in the published structures [6,32]. In addition, two specific anti-ganglioside antibodies were also included, one anti- $\mathrm{G}_{\mathrm{M} 1}$ and one anti- $\mathrm{G}_{\mathrm{M} 2}$ (Figure 2).

Table 2 summarises the binding results obtained using our lectin array for both purified (11168 [P]) and crudely isolated (11168 [C]) LOS preparations. Even though binding of BODIPY labelled LOS to all lectins/antibodies with the exception of DBA, ConA and CTB was observed, some differences with respect to the level of binding was 
observed (Table 2; Figure 3). That is, three lectins were found to bind both LOS preparations down to $125 \mu \mathrm{g} / \mathrm{mL}$ (lowest concentration printed), the $\beta$-Gal recognising lectins PNA and VAA, and the $\alpha-G a l$ recognising Jacalin (structures recognised shown in Figure 2). The lack of LOS binding to DBA and ConA was anticipated, however the inability of either LOS preparation to binding immobilised CTB was surprising, particularly given that lectin blot analysis using HRPconjugated CTB showed significant binding (Figure 1B). Both antiganglioside antibodies were bound by both LOS preparations, however binding to anti- $\mathrm{G}_{\mathrm{M} 2}$ was only observed at the highest concentration printed, while anti-GM1 was bound down to the 1:1000 dilution (Table 2). This is not surprising given that the published C. jejuni LOS structure is known to be exclusively $G_{M 1}$ under specified growth conditions [35]. The low binding therefore observed to anti- $\mathrm{G}_{\mathrm{M} 2}$ may simply reflect some cross-specificity to the underlying $\mathrm{G}_{\mathrm{M} 2}$ structure within the $\mathrm{G}_{\mathrm{M} 1}$ mimic. In comparison, live C. jejuni 11168-O bound to all printed spots that contained protein including CTB (Figure 3C).

Further analysis was performed on LOS isolated using the crude method from other $C$. jejuni strains, one with a published LOS structure 81-176 (Structure shown in Figure 2) [32] and a strain without a published structure, C. jejuni 224. C. jejuni 224 was chosen for analysis as it has a LOS biosynthesis cluster (Class R; Table 3A) capable of producing molecular mimicry (Class A, B, C, R and M; Table 3B) $[28,29]$.

Lectin array analysis confirmed C. jejuni 81-176 produces LOS with $G_{M 2}$ mimicry rather than $G_{M 1}$. Binding was observed for the two highest concentrations of the Anti- $\mathrm{G}_{\mathrm{M} 2}$ antibody, but no binding was observed for $\mathrm{G}_{\mathrm{M} 1}$. The LOS from 81-176 also had decreased binding for PNA, Jacalin and VAA when compared to NCTC11168 further confirming the absence of the terminal galactose from the structure (Figure 2). Binding of the 81-176 LOS to LFA and MAA confirmed the presence of sialylation on the LOS.

Analysis of C. jejuni strain 224 revealed binding to the anti- $\mathrm{G}_{\mathrm{M} 1}$ antibody but other binding to structures lectins such as PNA, Jacalin and VAA were only equal in binding to those observed for 81-176 rather than NCTC11168. The LOS isolated from C. jejuni 224 was confirmed to be sialylated due to positive binding by LFA and MAA. No binding was observed for the antiG $\mathrm{M}_{\mathrm{M} 2}$ antibody.

\section{Discussion}

The use of lectin arrays to determine surface glycan structures on whole bacteria has been previously described $[1 ; 8]$; however, to our knowledge, our approach of analysing fluorescently labelled isolated LOS on lectin array is completely novel, and offers a powerful analytical technique. This is particular the case, because the use of whole bacteria on lectin arrays may not accurately reveal the nature of the glycosylation present on the cell. As previously stated, lectins and antibodies are glycoproteins [11,12], therefore carbohydrate recognising adhesins present on bacteria [13-17] may bind these structures, rather than immobilised lectins binding to bacterial surface glycoconjugates. Testing of whole $C$. jejuni found binding to all lectins printed including CTB which had failed to bind in all other experiments (Figure 3; Table 2). Our novel approach of utilising isolated LOS overcomes this potentially significant shortcoming. Using the C. jejuni 11168 LOS as a model our discrete lectin array identified all carbohydrate components (Table 2) as predicted from the published structure (Figure 2).

The success of our approach depended largely on the dye used to label the isolated LOS. The lipophilic BODIPY TR methyl ester specifically interacts with the hydrocarbon tail of the LOS. The lack of binding observed to both DBA and ConA on the array indirectly confirms this selective labelling, given that any contaminating glycoconjugates (eg. glycoproteins) co-isolated with the C. jejuni LOS that could have been labelled with BODIPY would be expected to bind DBA immobilised on our array. That is, terminal $\alpha$-D-GalNAc structures are common in N-linked C. jejuni glycoproteins [36], and if labelled, would have been detected on our lectin array.

Analysis was also performed on two other strains of C. jejuni for LOS molecular mimicry, 81-176 and 224. C. jejuni 81-176 was chosen because, like NCTC1168 [6], 81-176 has a published structure [32]. The results of the lectin array agreed with the known structure produced by $81-176$, a $\mathrm{G}_{\mathrm{M} 2}$ mimicking LOS [32]. C. jejuni 81-176 is known to produce several other structures including $G_{M 3}, G_{D 1 b}$ and $\mathrm{G}_{\mathrm{D} 2}$, however, these structures are present in smaller amounts than the $\mathrm{G}_{\mathrm{M} 2}$ mimicking structures [32]. Therefore it is unlikely these structures would be affecting the outcomes of the array analysis. A wider variety of anti-ganglioside antibodies may prove effective in identifying these less prevalent LOS structures.

C. jejuni $224 \mathrm{LOS}$ analysed by lectin array indicated primarily $\mathrm{G}_{\mathrm{MI}}$ mimicry from the antibody binding but was not $100 \%$ confirmed by the binding observed for the other lectins present on the array. Levels of binding for PNA, VAA and Jacalin were lower than those seen for the known $\mathrm{G}_{\mathrm{M} 1}$ mimic NCTC11168 (Table 2). However, the LOS structure produced by $C$. jejuni 224 was definitely sialylated providing further evidence for gangloside mimicry. A previous study showed that the LOS from C. jejuni 224 was of the same size by electrophoresis as NCTC11168 and bound strongly by CTB [35]. This result together with the lectin array result implicates strongly that the $\mathrm{LOS}$ is a $\mathrm{G}_{\mathrm{M} 1}$ mimic.

Although our lectin array data correlates well with the known C. jejuni 11168 and 81-176 LOS structures, a discrepancy between observed and expected binding results was noted. Specifically, no binding was observed to CTB immobilised on the lectin array, even though lectin blotting using CTB (Figure 1) showed a strong positive signal. This was a predictable outcome since CTB exists as a pentameric structure [37]. The complex pentameric structure of CTB may therefore be disrupted or constrained when covalently attached to the array through an epoxside-linkage. CTB is likely to be unsuitable for use on lectin arrays and its use restricted to lectin blotting analyses.

We also investigated the suitability of two different LOS preparations (phenol-purified and crudely isolated LOS) for analysis on our lectin array. Apart from a slight increase in non-specific background binding of the crude LOS preparation, compared to the phenol purified LOS (data not shown), no significant difference was observed using either LOS preparations. This suggests that a simple heat lysis and proteinase $\mathrm{K}$ digestion of $C$. jejuni or other bacteria is sufficient to allow rapid and sensitive screening using lectin arrays particularly of strains expressing ganglioside mimicry. The simple methodology reported here can, therefore, be used to rapidly evaluate whether clinical isolates have the potential to produce adverse autoimmune reactions as post infection sequelae, similar to those attributed to C. jejuni (GBS or MFS). Knowing whether clinically important bacterial strains are capable of inducing severe autoimmune responses may aid in prevention and/or early diagnosis of these debilitating post infection conditions.

The method reported here is also applicable, through minor modifications to the lectins/antibody specificities printed on the array, to the screen glycolipids from almost any other species of bacteria for almost any terminal glycoconjugate. 
Citation: Semchenko EA, Moutin M, Korolik V, Tiralongo J, Day CJ (2011) Lectin Array Analysis of Purified Lipooligosaccharide: A Method for the Determination of Molecular Mimicry. J Glycom Lipidom 1:105. doi:10.4172/2153-0637.1000105

Page 5 of 5

\section{References}

1. Hsu KL, Pilobello KT, Mahal LK (2006) Analyzing the dynamic bacterial glycome with a lectin microarray approach. Nat Chem Biol 2: 153-157.

2. Gilbert M, Brisson JR, Karwaski MF, Michniewicz J, Cunningham AM, et al (2000) Biosynthesis of ganglioside mimics in Campylobacter jejuni OH4384 Identification of the glycosyltransferase genes, enzymatic synthesis of model compounds, and characterization of nanomole amounts by $600-\mathrm{mhz}(1) \mathrm{h}$ and (13)c NMR analysis. J Biol Chem 275: 3896-3906.

3. Gilbert M, Parker CT, Moran AP (2008) Campylobacter jejuni lipooligosaccharides: structures and biosynthesis ASM Press Washington, DC.

4. Moran AP, Annuk H, Prendergast MM (2005) Antibodies induced by ganglioside-mimicking Campylobacter jejuni lipooligosaccharides recognise epitopes at the nodes of Ranvier. J Neuroimmunol 165: 179-185.

5. Peak IR, Grice ID, Faglin I, Klipic Z, Collins PM, et al. (2007) Towards understanding the functional role of the glycosyltransferases involved in the biosynthesis of Moraxella catarrhalis lipooligosaccharide. Febs J 274: 2024 2037.

6. St Michael F, Szymanski CM, Li J, Chan KH, Khieu NH, et al. (2002) The structures of the lipooligosaccharide and capsule polysaccharide of Campylobacter jejuni genome sequenced strain NCTC 11168. Eur J Biochem. 269: 5119-5136.

7. Fogg GC, Yang LY, Wang E, Blaser MJ (1990) Surface array proteins of Campylobacter fetus block lectin-mediated binding to type A lipopolysaccharide. Infect Immun 58: 2738-2744.

8. Hsu KL, Mahal LK (2006) A lectin microarray approach for the rapid analysis of bacterial glycans. Nat Protoc 1: 543-549.

9. Tao SC, Li Y, Zhou J, Qian J, Schnaar RL, et al. (2008) Lectin microarrays identify cell-specific and functionally significant cell surface glycan markers. Glycobiology 18: 761-769.

10. Tateno H, Uchiyama N, Kuno A, Togayachi A, Sato T, et al. (2007) A novel strategy for mammalian cell surface glycome profiling using lectin microarray. Glycobiology 17: 1138-1146.

11. Ashford DA, Dwek RA, Rademacher TW, Lis H, Sharon N (1991) The glycosylation of glycoprotein lectins. Intra- and inter-genus variation in $\mathrm{N}$-linked oligosaccharide expression. Carbohydr Res 213: 215-227.

12. Debray H, Wieruszeski JM, Strecker G, Franz H (1992) Structural analysis of the carbohydrate chains isolated from mistletoe (Viscum album) lectin I. Carbohydr Res 236: 135-143

13. Aspholm-Hurtig M, Dailide G, Lahmann M, Kalia A, Ilver D, et al. (2004) Functional adaptation of $\mathrm{BabA}$, the $\mathrm{H}$. pylori $\mathrm{ABO}$ blood group antigen binding adhesin. Science 305: 519-522.

14. Gilboa-Garber N, Sudakevitz D (1999) The hemagglutinating activities of Pseudomonas aeruginosa lectins PA-IL and PA-IIL exhibit opposite temperature profiles due to different receptor types. FEMS Immunol Med Microbiol 25: 365-369.

15. Lehmann F, Tiralongo E, Tiralongo J (2006) Sialic acid-specific lectins: occurrence, specificity and function. Cell Mol Life Sci 63: 1331-1354.

16. Thomas WE, Nilsson LM, Forero M, Sokurenko EV, Vogel V (2004) Sheardependent 'stick-and-roll' adhesion of type 1 fimbriated Escherichia coli. Mol Microbiol 53: 1545-1557.

17. Thomas WE, Trintchina E, Forero M, Vogel V, Sokurenko EV (2002) Bacteria adhesion to target cells enhanced by shear force. Cell 109: 913-923.

18. Szymanski CM, Logan SM, Linton D, Wren BW (2003) Campylobacter--a tale of two protein glycosylation systems. Trends Microbiol 11: 233-238.

19. Nachamkin I, Allos BM, Ho T (1998) Campylobacter species and Guillain-Barre syndrome. Clin Microbiol Rev 11: 555-567.

20. Prendergast MM, Moran AP (2000) Lipopolysaccharides in the development of the Guillain-Barre syndrome and Miller Fisher syndrome forms of acute inflammatory peripheral neuropathies. J Endotoxin Res 6: 341-359.

21. Yuki N, Susuki K, Koga M, Nishimoto Y, Odaka M, et al. (2004) Carbohydrate mimicry between human ganglioside GM1 and Campylobacter jejun lipooligosaccharide causes Guillain-Barre syndrome. Proc Natl Acad Sci U S A 101: 11404-11409.

22. Gaynor EC, Cawthraw S, Manning G, MacKichan JK, Falkow S, et al. (2004)
The genome-sequenced variant of Campylobacter jejuni NCTC 11168 and the original clonal clinical isolate differ markedly in colonization, gene expression, and virulence-associated phenotypes. J Bacteriol 186: 503-517.

23. Hitchcock PJ, Brown TM (1983) Morphological heterogeneity among Salmonella lipopolysaccharide chemotypes in silver-stained polyacrylamide gels J Bacteriol. 154: 269-277.

24. Westphal O, Luderitz O, Bister O (1952) Uber die Extraktion von Bakterien mit Phenol/Wasser Naturforsch. 7: 148-155.

25. Moran AP, Helander IM, Kosunen TU (1992) Compositional analysis of Helicobacter pylori rough-form lipopolysaccharides J Bacteriol. 174: 13701377.

26. H Schagger (2006) Tricine-SDS-PAGE Nat Protoc. 1: 16-22.

27. Tsai CM, Frasch CE (1982) A sensitive silver stain for detecting lipopolysaccharides in polyacrylamide gels Anal Biochem. 119: 115-119.

28. Parker CT, Gilbert M, Yuki N, Endtz HP, Mandrell RE (2008) Characterization of lipooligosaccharide-biosynthetic loci of Campylobacter jejuni reveals new lipooligosaccharide classes: evidence of mosaic organizations J Bacteriol. 190: 5681-5689.

29. Parker CT, Horn ST, Gilbert M, Miller WG, Woodward DL, et al. (2005) Comparison of Campylobacter jejuni lipooligosaccharide biosynthesis loci from a variety of sources J Clin Microbiol. 43: 2771-2781.

30. Day CJ, Tiralongo J, Hartnell RD, Logue CA, Wilson JC, et.al (2009) Differential carbohydrate recognition by Campylobacter jejuni strain 11168: influences of temperature and growth conditions PLoS One 4: e4927.

31. Hartley-Tassell LE, Shewell LK, Day CJ, Wilson JC, Sandhu R, et al. (2010) Identification and characterization of the aspartate chemosensory receptor of Campylobacter jejuni Mol Microbiol. 75: 710-730.

32. Guerry P, Szymanski CM, Prendergast MM, Hickey TE, Ewing CP, et al. (2002) Phase variation of Campylobacter jejuni 81-176 lipooligosaccharide affects ganglioside mimicry and invasiveness in vitro Infect Immun. 70: 787-793.

33. Karlyshev AV, Ketley JM, Wren BW (2005) The Campylobacter jejuni glycome. FEMS Microbiol Rev 29: 377-390.

34. Linton D, Gilbert M, Hitchen PG, Dell A, Morris HR, et al. (2000) Phase variation of a beta-1,3 galactosyltransferase involved in generation of the ganglioside GM1-like lipo-oligosaccharide of Campylobacter jejuni Mol Microbiol 37: 501 514

35. Semchenko EA, Day CJ, Wilson JC, Grice ID, Moran AP et al. (2010) Temperature-dependent phenotypic variation of Campylobacter jejun lipooligosaccharides BMC Microbiol. 10: 305

36. Young NM, Brisson JR, Kelly J, Watson DC, Tessier L, et al. (2002) Structure of the N-linked glycan present on multiple glycoproteins in the Gram-negative bacterium, Campylobacter jejuni J Biol Chem. 277: 42530-42539.

37. Zhang RG, Westbrook ML, Westbrook EM, Scott DL, Otwinowski Z, et al. (1995) The $2.4 \mathrm{~A}$ crystal structure of cholera toxin B subunit pentamer: choleragenoid. J Mol Biol 251: 550-562.

\section{Submit your next manuscript and get advantages of OMICS Group submissions}

Unique features:

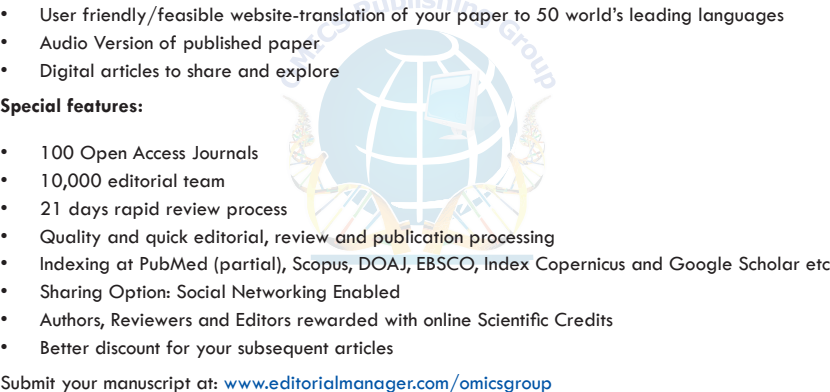

